\title{
Excitation modes of ${ }^{6} \mathrm{He}$ from proton collisions
}

\author{
R. Crespo* \\ Departamento de Física, Instituto Superior Técnico, Taguspark, Avenida Professor Cavaco Silva, Taguspark, \\ 2780-990 Porto Salvo, Oeiras, Portugal \\ I. J. Thompson ${ }^{\dagger}$ \\ Department of Physics, University of Surrey, Guildford, Surrey GU2 7XH, United Kingdom \\ A. M. Moro \\ Departmento de FAMN, Facultad de Física, Universidad de Sevilla, Apartado 1065, E-41080, Sevilla, Spain
}

(Received 27 July 2006; published 31 October 2006)

\begin{abstract}
The cross section for inelastic scattering of Borromean halo nuclei from protons is evaluated using the Multiple Scattering expansion of the Total transition amplitude (MST) formalism. Differential cross sections and energy spectra are shown for $p-{ }^{6} \mathrm{He}$ at $700 \mathrm{MeV} /$ nucleon. The role of spin flip excitations is analyzed.

DOI: 10.1103/PhysRevC.74.044616

PACS number(s): $24.10 . \mathrm{Ht}, 25.40 . \mathrm{Cm}$
\end{abstract}

\section{INTRODUCTION}

The Borromean two-neutron halo nuclei ${ }^{11} \mathrm{Li}$ and ${ }^{6} \mathrm{He}$ have been attracting many theoretical and experimental studies since the ground state and the excited modes are not both well understood yet, and knowledge of the continuum properties will contribute to our understanding of the properties of these nuclei. To illustrate the puzzling features half-unveiled in this field, various theories [1-3] predict a variety of excitation/resonance modes at low energies in the continuum of the above Borromean nuclei, but up to now there is only a clear evidence of a $J^{\pi}=2^{+}$resonance for ${ }^{6} \mathrm{He}$ [4]. Thus, for more than a decade the existence and the understanding of the excitation modes is far from being achieved, now a detailed study of the resonances in the continuum sea is one of the priority tasks of radioactive beam research.

From a theoretical point of view, structure calculations predict a rich continuum. Within few body cluster models that include the three-body dynamics of the Borromean system [5], two kinds of phenomena have been predicted: (i) true three-body resonances, with three-body phase shifts crossing $\pi / 2$ in all partial waves, and (ii) not-fully-fledged resonant contributions with fast growing three-body phase shifts up to $\pi / 2$ in some partial waves. These few-body cluster models rely on fitted effective interactions between the cluster constituents. In addition, the simultaneous treatment of the Pauli principle and continuum pairing in these approaches remains today an open problem. Shell model approaches have also been developed but the coupling to the continuum presents a difficult problem. Most often, excited states may appear to be resonances in some structure models, in particular for the soft dipole mode in Borromean nuclei. This calls for an improvement of the structure approaches, and for new work to test the structure theories by means of reaction observables.

\footnotetext{
*Electronic address: Raquel.Crespo@tagus.ist.utl.pt

${ }^{\dagger}$ Electronic address: I.Thompson@surrey.ac.uk

Electronic address: moro@us.es
}

The low lying excited states of the Borromean nuclei have also been experimentally investigated. These very difficult studies have also often led to some contradictory results in particular with respect to existence of a dipole excitation [6]. It is thus fair to say that our theoretical and experimental knowledge of the Borromean resonant continuum sea is today still very inconclusive.

Inelastic scattering may be a useful process to study the excitation modes of halo nuclei, if it is possible to single out particular multipole excitations. Recently the excitation modes of ${ }^{11} \mathrm{Li}$ were studied from inelastic collisions with protons making use of the Multiple Scattering expansion of the total Transition amplitude (MST) [7]. In this work good agreement with the experimental data [8] was obtained with a structure model in which there is a pronounced $1^{-}$peak at low continuum energies but yet in which there is not a fully-fledged resonance in this breakup channel.

Excitation of the ${ }^{6} \mathrm{He}$ continuum by inelastic collisions from protons has been predicted using a distorted wave few-body method [9-11]. This method has the disadvantage of the ambiguity in the choice of the optical potential for the final excited states. The calculated inelastic observables show the contribution from the first well known $2_{1}^{+}$low energy resonance. The predicted higher energy spectrum results from a composition of overlapping soft modes of multipolarities $1^{-}, 2_{2}^{+}, 1^{+}, 0_{2}^{+}$.

We present here a study of inelastic scattering of ${ }^{6} \mathrm{He}$ from protons within a few body picture description of this nucleus, assumed well described by a core and two valence neutrons.

We shall review the structure approach, namely the method used to obtain the bound and scattering solutions of a threebody problem. At the same time we shall describe in detail the scattering framework, that is, the Multiple Scattering expansion of the total Transition amplitude (MST) [12], which explicitly incorporates the halo degrees of freedom. We shall make a study of the calculated observables using different interactions between the few-body clusters. In addition the role of spin interactions in the excitation will be analyzed. 


\section{SCATTERING FORMALISM}

The halo degrees of freedom can be explicitly incorporated in the scattering framework in a convenient way within the MST approach [7,12].

We consider then the scattering of a nucleon (particle 1) from a weakly bound system of $\mathcal{N}$ subsystems. The total transition amplitude $T$ can be written as a multiple scattering expansion in the transition amplitudes $\hat{t}_{\mathcal{I}}$ for proton scattering from each projectile subsystem $\mathcal{I}$ [12]

$$
\mathcal{T}=\sum_{\mathcal{I}} \hat{t}_{1 \mathcal{I}}+\sum_{\mathcal{I}} \hat{t}_{1 \mathcal{I}} G_{0} \sum_{\mathcal{J} \neq \mathcal{I}} \hat{t}_{1 \mathcal{J}}+\cdots,
$$

where the proton $-\mathcal{I}$ subsystem transition amplitude satisfies

$$
\hat{t}_{1 \mathcal{I}}=v_{1 \mathcal{I}}+v_{1 \mathcal{I}} G_{0} \hat{t}_{1 \mathcal{I}},
$$

with $v_{1 \mathcal{I}}$ the interaction between the nucleon and $\mathcal{I}$ subsystem (here the core and valence neutrons). The propagator $G_{0}=$ $\left(E^{+}-K\right)^{-1}$, within the impulse approximation, contains the kinetic energy operators of the projectile and all the target subsystems $[13,14]$. Here $E$ is the kinetic energy, $E=\frac{\hbar^{2} k^{2}}{2 \mu_{N A}}$ in the overall center of mass frame, and $\mu_{N A}$ is the protonprojectile reduced mass. As follows from Eq. (1), in the MST expansion the few-body dynamics is properly included, and excitations of the nucleus which involve changes in the relative motion of the subsystems are taken into account. The contribution of these to the calculated elastic scattering cross section was investigated in Refs. [12,15].

We are interested here in the study of Borromean halo systems assumed well described by a three-body model of two valence nucleons (labeled 2 and 3 ) and a core (labeled 4) in a bound state at energy $\epsilon_{0}$. Within the single scattering approximation, as will be used here, the total transition amplitude for the scattering of a nucleon from the Borromean nucleus system Eq. (1) reduces to

$$
\mathcal{T}=\sum_{\mathcal{I}=2,4} \hat{t}_{1 \mathcal{I}}
$$

Energy conservation relates the scattered momentum $k$ of excited states at continuum energy $\epsilon$ as

$$
\frac{\hbar^{2}}{2 \mu_{N A}} k^{2}+\epsilon=\frac{\hbar^{2}}{2 \mu_{N A}} k_{0}^{2}+\epsilon_{0} .
$$

\section{STRUCTURE}

\section{A. The solution of the three-body problem}

For completeness we describe here the hyperspherical harmonics $(\mathrm{HH})$ method used to describe the ground state and the continuum states of the two-neutron (three-body) Borromean systems, such as ${ }^{6} \mathrm{He}$ represented in Fig. 1. The total wave function, $\Phi_{\epsilon}^{J M}$ satisfies

$$
\left(\hat{T}+\hat{V}+\hat{V}_{\text {core }}-\epsilon\right) \Phi_{\epsilon}^{J M}=0,
$$

where $\hat{V}=\sum_{\mathcal{I}} \sum_{\mathcal{J} \neq \mathcal{I}} \hat{V}_{\mathcal{I} \mathcal{J}}+\hat{V}^{3 \mathrm{~B}}$ includes binary interactions between the clusters $\hat{V}_{\mathcal{I} \mathcal{J}}$ and also a possible effective three-body potential, $\hat{V}^{3 \mathrm{~B}}$, that takes into account other closed channels.
Assuming the core is inert and spinless, the three-body bound state and continuum wave functions can be written as

$$
\Phi_{\epsilon}^{J M}\left(\vec{r}, \vec{R}, \vec{\xi}_{c}\right)=\varphi_{\epsilon}^{J M}(\vec{r}, \vec{R}) \otimes \varphi_{\text {core }}\left(\vec{\xi}_{c}\right),
$$

where $\varphi_{\text {core }}\left(\vec{\xi}_{c}\right)$ is the core internal wave function and $\varphi_{\epsilon}^{J M}(\vec{r}, \vec{R})$ the three-body valence wave function relative to the core either bound or in the continuum, that in Jacobi coordinates can be written in the general form

$$
\varphi_{\epsilon}^{J M}(\vec{r}, \vec{R})=\sum_{\gamma} \mathcal{F}_{\gamma}^{J \epsilon}(r, R) \widehat{Y}_{\gamma}^{J M}(\Omega),
$$

with $\Omega=\{\hat{r}, \hat{R}\}, \gamma=\left\{\ell_{x} \ell_{y} L S\right\}$,

$$
\widehat{Y}_{\gamma}^{J M}(\Omega)=\left\{\left[Y_{\ell_{x}}(\hat{r}) \otimes Y_{\ell_{y}}(\hat{R})\right]_{L} \otimes\left[\chi_{s_{2}} \otimes \chi_{s_{3}}\right]_{S}\right\}_{J M},
$$

and $\chi_{s}$ the neutron spin functions.

However, for the purpose of evaluating the wave functions, the nature of the Borromean system makes it convenient to introduce a set hyper-radial coordinates: the hyperradius $\rho$ and five hyperspherical polar angles $\Omega_{5}=\left\{\alpha, \theta_{x}, \phi_{x}, \theta_{y}, \phi_{y}\right\}$. The hyper-radius $\rho$ is defined as $\rho=\sqrt{x^{2}+y^{2}}$ with scaled coordinates $\vec{x}=2^{-1 / 2} \vec{r}$ from the relative coordinate between the valence nucleons, and $\vec{y}=\left(2 A_{c} /\left(2+A_{c}\right)\right)^{1 / 2} \vec{R}$ from the relative to the core position of the neutron pair. The angle $\alpha=\arctan (x / y)$ is the hyperangle and $\theta_{x}, \phi_{x}, \theta_{y}, \phi_{y}$ the angles associated with the unit spatial vectors $\hat{x}$ and $\hat{y}$.

The hyperspherical harmonics $(\mathrm{HH})$ method expands both the bound and continuum wave functions using hyperspherical harmonic basis functions [16]

$$
\Upsilon_{\mathcal{K}_{\gamma}}^{J M}\left(\Omega_{5}\right)=\left\{\mathcal{Y}_{{\mathcal{K} \ell_{x} \ell_{y} L}}\left(\Omega_{5}\right) \otimes\left[\chi_{s_{2}} \otimes \chi_{s_{3}}\right]_{S}\right\}_{J M},
$$

with

$$
\mathcal{Y}_{\mathcal{K} \ell_{x} \ell_{y} L M_{L}}\left(\Omega_{5}\right)=\psi_{\mathcal{K} \ell_{x} \ell_{y}}(\alpha)\left[Y_{\ell_{x}}(\hat{x}) \otimes Y_{\ell_{y}}(\hat{y})\right]_{L M_{L}} .
$$

The quantum number $\mathcal{K}=\ell_{x}+\ell_{y}+2 n(n=0,1,2, \ldots)$ is called hyperangular momentum. The function $\psi_{\mathcal{K} \ell_{x} \ell_{y}}(\alpha)$, has an explicit form in terms of Jacobi polynomials of the hyperangle $\alpha$ [16]. The HH exploits the feature that the wave function tends to be concentrated in only a few components which correspond to the lowest hyperangular momenta of the system.

The three-body valence wave function relative to the core has then the general form

$$
\varphi_{\epsilon}^{J M}(\vec{r}, \vec{R})=\rho^{-5 / 2} \sum_{\mathcal{K} \gamma} F_{\mathcal{K} \gamma}^{J \epsilon}(\rho) \Upsilon_{\mathcal{K}_{\gamma}}^{J M}\left(\Omega_{5}\right) .
$$

The radial wave functions $F_{\mathcal{K} \gamma}^{J \epsilon}(\rho)$ are found by solving a coupled channels Schrödinger problem with a three-body effective centrifugal barrier $V_{\mathcal{L}}^{\text {centrif }}(\rho)$ and coupling potentials $V_{\mathcal{K}^{\prime} \gamma^{\prime}, \mathcal{K} \gamma}^{\text {coup }}(\rho)$. The centrifugal barrier

$$
V_{\mathcal{L}}^{\text {centrif }}(\rho)=\frac{\hbar^{2}}{2 m} \frac{\mathcal{L}(\mathcal{L}+1)}{\rho^{2}},
$$

with $\mathcal{L}=\mathcal{K}+3 / 2$, is generated by the hyperangular momentum $\mathcal{K}$ and does not vanish even if the angular momentum $\ell_{y}$ and $\ell_{x}$ of the subsystems are equal to zero. The coupling potentials take into account all the interactions between the 
bodies $\mathcal{I}$ and $\mathcal{J}$ and the effective 3B interaction

$$
\begin{aligned}
V_{\mathcal{K}^{\prime} \gamma^{\prime}, \mathcal{K} \gamma}^{\text {coup }}(\rho)= & \left\langle\Upsilon_{\mathcal{K}_{\gamma}}\left(\Omega_{5}\right)\left|\sum_{\mathcal{I}} \sum_{\mathcal{J} \neq \mathcal{I}} V_{\mathcal{I} \mathcal{J}}\left(\rho, \Omega_{5}\right)\right| \Upsilon_{\mathcal{K}^{\prime} \gamma^{\prime}}\left(\Omega_{5}\right)\right\rangle \\
& +\left\langle\Upsilon_{\mathcal{K}_{\gamma}}\left(\Omega_{5}\right)\left|V^{3 \mathrm{~B}}\left(\rho, \Omega_{5}\right)\right| \Upsilon_{\mathcal{K}^{\prime} \gamma^{\prime}}\left(\Omega_{5}\right)\right\rangle .
\end{aligned}
$$

It follows from Eqs. (7) and (11) that the radial wave functions in Jacobi coordinates can be obtained from the radial wave functions in hyperspherical coordinates according to

$$
\mathcal{F}_{\gamma}^{J \epsilon}(r, R)=\sum_{\mathcal{K}} \rho^{-5 / 2} \psi_{\mathcal{K} \ell_{x} \ell_{y}}(\alpha) F_{\mathcal{K} \gamma}^{J \epsilon}(\rho) .
$$

There are two methods of finding the solution of coupled equations which differ essentially in the asymptotic behavior of the continuum wave functions: the scattering method and the pseudostates method.

\section{The scattering method}

Within this approach, the appropriate boundary conditions of the three-body wave function are introduced. The bound state at energy $\epsilon_{0}$ has the asymptotic behavior such that

$$
\lim _{\rho \rightarrow \infty} F_{\mathcal{K} \gamma}^{J \epsilon_{0}}(\rho) \rightarrow 0
$$

and the continuum wave functions are written as

$$
\begin{aligned}
\varphi_{\epsilon, m_{1} m_{2}}\left(\vec{x}, \vec{y}, \hat{\mathbf{k}}_{x}, \hat{\mathbf{k}}_{y}, \alpha^{\kappa}\right)= & (\kappa \rho)^{-5 / 2} \\
& \times \sum_{J M \mathcal{K} \mathcal{K}^{\prime} \gamma \gamma^{\prime}} F_{\mathcal{K}^{\prime} ; \mathcal{K}^{\prime} \gamma^{\prime}}^{J}(\kappa \rho) \Upsilon_{\mathcal{K}^{\prime} \gamma}^{J M}\left(\Omega_{5}\right) \\
& \times \sum_{M_{L} M_{S}}\left\langle L M_{L} S M_{S} \mid J M\right\rangle \\
& \times\left\langle S_{2} m_{2} S_{3} m_{3} \mid S M_{S}\right\rangle \mathcal{Y}_{\mathcal{K} \ell_{x} \ell_{y} L}^{M_{L}}\left(\Omega_{5}^{\kappa}\right),
\end{aligned}
$$

where $m_{1}$ and $m_{2}$ are the initial asymptotic spin projections, $\hbar^{2} \kappa^{2} / 2 m=\epsilon$ and $\tan \alpha^{\kappa}=k_{x} / k_{y}$. The radial part of the wave function has the asymptotic normalization of an incoming and outgoing wave in the hyper-radius

$$
\lim _{\rho \rightarrow \infty} F_{\mathcal{K} \gamma ; \mathcal{K}^{\prime} \gamma^{\prime}}^{J}(\kappa \rho)=\frac{i}{2} \delta_{\Lambda \Lambda^{\prime}} \exp (-i \kappa \rho)-\frac{i}{2} S_{\Lambda ; \Lambda^{\prime}} \exp (i \kappa \rho),
$$

with $\Lambda=\{\mathcal{K}, \gamma\}$ and where $S_{\Lambda ; \Lambda^{\prime}}$ is the S-matrix for the $3 \rightarrow 3$ scattering for an incoming wave in the channel $\mathcal{K}^{\prime} \gamma^{\prime}$. The continuum functions are then obtained either by using radial integration of coupled equations [17], or using R-matrix expansions [18].

\section{Pseudostates method}

An alternative approximate method, if only the interior part of the wave functions is required, and if some averaging over energy is permitted, is to solve the coupled equations by finding pseudostates in a hyper-radial box. This involves expanding the wave function with an square-integrable basis, and then diagonalizing the Hamiltonian matrix. To make the number of states finite, only eigenstates below a certain excitation energy are retained. Then the Hamiltonian is diagonalized in this truncated basis. The set of these energy eigenfunctions will be a good representation of continuum states at least in the interior region. For the present purposes we use the GaussLaguerre hyper-radial basis, $R_{n}(\rho)$,

$$
R_{n}(\rho)=\rho_{0}{ }^{-3}[n ! /(n+5) !]^{1 / 2} L_{n}^{5}(z) \exp (-z / 2)
$$

with $z=\rho / \rho_{0}$, orthonormalized such that

$$
\int_{0}^{\infty} d \rho \rho^{5} R_{n}(\rho) R_{n^{\prime}}(\rho)=\delta_{n n^{\prime}}
$$

and $\rho_{0}$ a parameter to set the radial scale of the basis.

The eigenstates for positive energies $\epsilon_{i}$ are then the discrete quasibound states, $\hat{\varphi}_{\epsilon_{i}}^{J M}(\vec{x}, \vec{y})$

$$
\hat{\varphi}_{\epsilon_{i}}^{J M}(\vec{x}, \vec{y})=\rho^{-5 / 2} \sum_{\mathcal{K} \gamma} \hat{F}_{\mathcal{K} \gamma}^{J \epsilon_{i}}(\rho) \Upsilon_{\mathcal{K}_{\gamma}}^{J M}\left(\Omega_{5}\right)
$$

with

$$
\hat{F}_{\mathcal{K}_{\gamma}}^{J \epsilon_{i}}(\rho)=\rho^{5 / 2} \sum_{n=1}^{N} c_{n \mathcal{K} \gamma}^{J \epsilon_{i}} R_{n}(\rho)
$$

The width associated with each pseudo-state can be calculated following the methods in Refs. $[19,20]$ giving

$$
\Gamma^{J \epsilon_{i}}=2 \pi \frac{\hbar^{2}}{2 m} \sum_{\mathcal{K} \gamma}\left|B_{\mathcal{K}_{\gamma}}^{J \epsilon_{i}}\right|^{2}
$$

with the decay amplitudes given by

$$
B_{\mathcal{K} \gamma}^{J \epsilon_{i}}=\sum_{M}\left\langle\xi_{\epsilon_{i} \mathcal{K} \gamma}^{J M}\left(\rho, \Omega_{5}\right)\left|V_{24}+V_{34}\right| \hat{\varphi}_{\epsilon_{i}}^{J M}(\vec{x}, \vec{y})\right\rangle,
$$

where

$$
\xi_{\epsilon_{i} \mathcal{K} \gamma}^{J M}\left(\rho, \Omega_{5}\right)=\rho^{-2} J_{\mathcal{K}+2}\left(\kappa_{i} \rho\right) \Upsilon_{\mathcal{K}_{\gamma}}^{J M}\left(\Omega_{5}\right)
$$

with the momentum $\kappa_{i}$ given by $\hbar^{2} \kappa_{i}^{2} / 2 m=\epsilon_{i}$.

\section{B. The potentials}

In these few-body nuclear models, the needed interactions are those between the valence neutrons and the valence neutron and the core. Between the neutrons, we use the GPT $n-n$ potential [21] with spin-orbit and tensor components and we take the $n-{ }^{4} \mathrm{He}$ core potential from Ref. [22]. In order to overcome the underbinding of ${ }^{6} \mathrm{He}$ caused by omitting other closed channels, most important of which is the $t+t$, an additional effective three-body $\left(V_{J}^{3 \mathrm{~B}}\right)$ interaction is introduced. We consider two different forms of this potential. The first is the model (R5) of Ref. [16] with matrix elements of the form

$$
V_{\Lambda^{\prime}, \Lambda}^{3 \mathrm{~B}}(\rho)=\frac{\delta_{\Lambda^{\prime} \Lambda} V_{J}^{3 \mathrm{~B}}}{1+(\rho / 5)^{3}},
$$

and the second is model (R2) of Ref. [23] with matrix elements

$$
U_{\Lambda^{\prime}, \Lambda}^{3 \mathrm{~B}}(\rho)=\delta_{\Lambda^{\prime} \Lambda} U_{J}^{3 \mathrm{~B}} \exp \left(-\left(\rho / \rho_{0}\right)^{3}\right) .
$$

The $J=0$ strength of this effective potential is tuned to reproduce the experimental three-body separation energy, with $\mathrm{V}_{0}^{3 \mathrm{~B}}=-1.60 \mathrm{MeV}$ and $\mathrm{U}_{0}^{3 \mathrm{~B}}=-293.5 \mathrm{MeV}$ with $\rho_{0}=1 \mathrm{fm}$, and the $J>0$ strength tuned to give the $2_{1}^{+}$ 
resonance at the experimental energy: $\mathrm{V}_{J}^{3 \mathrm{~B}}=-0.85 \mathrm{MeV}$ and $\mathrm{U}_{J}^{3 \mathrm{~B}}=-293.5 \mathrm{MeV}$ with $\rho_{0}=1.25 \mathrm{fm}$. The first model (R5) predicts a two-neutron separation energy of $\mathrm{S}_{2 n}=0.975 \mathrm{MeV}$ and, with an $\alpha$ particle rms matter radius of $1.49 \mathrm{fm}$, yields a ${ }^{6} \mathrm{He}$ rms matter radius of $2.50 \mathrm{fm}$. Model (R2) predicts a similar $\mathrm{S}_{2 n}$, but produces a ${ }^{6} \mathrm{He}$ nucleus with a much smaller rms matter radius of $2.35 \mathrm{fm}$.

\section{The Borromean continuum sea}

Following Ref. [16], we define a fully fledged resonance when the phase shifts rise rapidly and cross $\pi / 2$ in at least one partial wave. These are in general associated with pockets in the corresponding diagonal interaction. The wave function behaves approximately in the interior for all dominant components as

$$
\hat{F}_{\mathcal{K} \gamma}^{J \epsilon}(\rho) \sim \mathcal{C}^{J \epsilon} \hat{\mathcal{F}}_{\mathcal{K} \gamma}^{J}(\rho)
$$

for a resonance at energy $\epsilon_{R}$, with the Breit-Wigner form

$$
\left|\mathcal{C}^{J \epsilon}\right|^{2}=\frac{\Gamma^{J \epsilon_{R}}}{\left(\epsilon-\epsilon_{R}\right)^{2}+\left[\Gamma^{J \epsilon_{R}}\right]^{2} / 4} .
$$

We also define a resonance like excitation when the phase shifts rise rapidly above threshold but not cross $\pi / 2$ in any of the partial waves. These are often caused by resonant and/or virtual systems in binary subsystems.

Both the dynamical models here considered (R2 and R5) reveal, in the continuum sea up to the ${ }^{3} \mathrm{H}+{ }^{3} \mathrm{H}$ threshold, the well known $J^{\pi}=2_{1}^{+}$, resonance and also a variety of other excitation modes with excitation energies slightly shifted: a broad resonance $J^{\pi}=1^{+}$, and resonance like excitations at $J^{\pi}=1^{-}, 0_{2}^{+}, 0^{-}$. The characteristics of the excitations/resonance states are summarized in Table I.

The $J^{\pi}=1^{-}$excitation has a dominant component $\left|\mathcal{K}\left[\ell_{x} \ell_{y}\right] L S\right\rangle=|1[01] 10\rangle$, which corresponds to a spatially extended dineutron in the $n n \mathrm{~S}$-wave with $\mathrm{P}$ motion relative to the core. The strong $n n$ attraction in the relative $S$-wave is responsible for the accumulation of the excitation strength at low continuum energies. The $J^{\pi}=0_{2}^{+}$is the first excitation, and the $J^{\pi}=2_{1}^{+}$is a narrow resonant state at energy $0.727 \mathrm{MeV}$ and width $\Gamma=0.058 \mathrm{MeV}$, with a dominant

\begin{tabular}{|c|c|c|c|c|}
\hline$J^{\pi}$ & $\mathcal{K}\left[\ell_{x} \ell_{y}\right] L, S$ & $\epsilon_{i}(\mathrm{MeV})$ & $\Gamma^{J \epsilon_{i}}(\mathrm{MeV})$ & $\%$ \\
\hline \multirow[t]{2}{*}{$0^{+}$} & $2\left[\begin{array}{ll}0 & 0\end{array}\right] 0,0$ & -0.957 & - & 83 \\
\hline & $2\left[\begin{array}{ll}1 & 1\end{array}\right] 1,1$ & & & 12 \\
\hline \multirow[t]{2}{*}{$0_{2}^{+}$} & $2\left[\begin{array}{ll}0 & 0\end{array}\right] 0,0$ & 1.095 & - & 95 \\
\hline & $2\left[\begin{array}{ll}1 & 1\end{array}\right] 1,1$ & & & 4 \\
\hline \multirow[t]{2}{*}{$0^{-}$} & $1\left[\begin{array}{ll}1 & 0\end{array}\right] 1,1$ & 1.765 & - & 84 \\
\hline & $1\left[\begin{array}{ll}1 & 2\end{array}\right] 1,1$ & & & 18 \\
\hline \multirow[t]{2}{*}{$1^{+}$} & $2\left[\begin{array}{ll}1 & 1\end{array}\right] 1,1$ & 1.787 & 1.1 & 86 \\
\hline & $2\left[\begin{array}{ll}1 & 1\end{array}\right] 0,1$ & & & 3 \\
\hline \multirow[t]{2}{*}{$1^{-}$} & $1\left[\begin{array}{ll}0 & 1\end{array}\right] 1,0$ & 1.030 & - & 72 \\
\hline & $1\left[\begin{array}{ll}2 & 1\end{array}\right] 1,0$ & & & 16 \\
\hline \multirow[t]{2}{*}{$2^{+}$} & $2\left[\begin{array}{ll}0 & 2\end{array}\right] 2,0$ & 0.727 & 0.058 & 45 \\
\hline & $1\left[\begin{array}{ll}1 & 1\end{array}\right] 1,1$ & & & 32 \\
\hline
\end{tabular}

TABLE I. ${ }^{6} \mathrm{He}$ ground state and excitation modes.
TABLE II. ${ }^{6} \mathrm{He}$ types of excitation.

\begin{tabular}{cccc}
\hline \hline$J^{\pi}$ & Type of excitation & Core contribution & Valence contribution \\
\hline $0_{2}^{+}$ & $\Delta S=0, \Delta L=0$ & $\mathrm{Y}$ & $\mathrm{Y}$ \\
$0^{-}$ & $\Delta S=1, \Delta L=0$ & $\mathrm{~N}$ & $\mathrm{Y}$ \\
$1^{+}$ & $\Delta S=1, \Delta L=1$ & $\mathrm{~N}$ & $\mathrm{Y}$ \\
$1^{-}$ & $\Delta S=0, \Delta L=1$ & $\mathrm{Y}$ & $\mathrm{Y}$ \\
$2^{+}$ & $\Delta S=0, \Delta L=2$ & $\mathrm{Y}$ & $\mathrm{Y}$ \\
\hline \hline
\end{tabular}

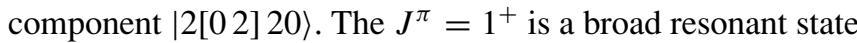
at energy $1.787 \mathrm{MeV}$ and width $\Gamma=1.1 \mathrm{MeV}$, with a dominant component $|2[11] 11\rangle$. It corresponds to a reorientation of the g.s. with the same quantum numbers.

The $0^{+} \rightarrow 0^{-}$and $0^{+} \rightarrow 1^{+}$excitations involve spin flip transitions and thus proceed through the scattering from valence nucleons as summarized in Table II. Therefore, these transitions are expected to give a small contribution to the scattering.

\section{THE REACTION FRAMEWORK}

Let us consider the general problem of the scattering of a nucleon (1) from a subsystem $\mathcal{I}$ with spin $\sigma_{\mathcal{I}}(0$ or $1 / 2)$.

The nucleon-subsystem $\mathcal{I}$ scattering amplitude can be conveniently described by the tensor representation [24]. In this representation, the matrix elements of the scattering amplitudes are written as

$$
\left\langle\vec{q}_{1 \mathcal{I}}^{\prime}\left|t_{1 \mathcal{I}}(\omega)\right| \vec{q}_{1 \mathcal{I}}\right\rangle=\sum_{\kappa q a b} t_{\kappa q}^{(a b)}\left(\omega, \vec{q}_{1 \mathcal{I}}^{\prime}, \vec{q}_{1 \mathcal{I}}\right) \mathcal{T}_{\kappa q}^{\dagger}(a, b),
$$

where $\mathcal{T}_{\kappa q}^{\dagger}(a, b)$ is a tensor of rank $\kappa$ given in terms of a linear combination for the spherical components of the spin operators of the two interacting systems:

$$
\mathcal{T}_{\kappa q}(a, b)=\sum_{\alpha \beta}(a \alpha b \beta \mid \kappa q) \tau_{a \alpha}\left(s_{1} ; 1\right) \tau_{b \beta}\left(s_{\mathcal{I}} ; \mathcal{I}\right) .
$$

In this equation $\tau_{a \alpha}\left(s_{1} ; 1\right)$ is the irreducible tensor operator for the projectile particle 1 with spin $s_{1}\left(a=0, \ldots 2 s_{1}\right) ; \tau_{b \beta}\left(s_{\mathcal{I}} ; \mathcal{I}\right)$ is the irreducible tensor operator for the struck subsystem $(\mathcal{I})$ with spin $s_{\mathcal{I}}\left(b=0, \ldots 2 s_{\mathcal{I}}\right)$. For nucleon scattering then since $s_{1}=\frac{1}{2}, \tau_{00}\left(\frac{1}{2} ; 1\right)=1$ and $\tau_{1 \beta}\left(\frac{1}{2} ; 1\right)=\sigma_{\beta}(1)$, with $\sigma_{\beta}(1)$ the spherical components of $\vec{\sigma}_{1}$ with respect to the chosen $z$-axis. We note that for a two body interacting system of spin $1 / 2$, the tensor of rank $\kappa=0$ can be obtained either when $a=b=0$ and $a=b=1$.

The transition amplitude $t_{\kappa q}^{(a b)}$ can be written in terms of the relative incoming and outgoing momenta $t_{\kappa q}^{(a b)}\left(\omega, \vec{q}_{1 \mathcal{I}}^{\prime}, \vec{q}_{1 \mathcal{I}}\right)$ or in terms of the transferred momentum $\overrightarrow{\mathcal{Q}}_{1 \mathcal{I}}=\vec{q}_{1 \mathcal{I}}^{\prime}-\vec{q}_{1 \mathcal{I}}$ and total momentum. On the energy shell then

$$
t_{\kappa q}^{(a b)}\left(\omega, \vec{q}_{1 \mathcal{I}}^{\prime}, \vec{q}_{1 \mathcal{I}}\right)=t_{\kappa q}^{(a b)}\left(\omega, \mathcal{Q}_{1 \mathcal{I}}\right)
$$

We consider now the scattering process of ${ }^{6} \mathrm{He}$, originally in a $\left|\Psi_{0}^{J_{0} M_{0}}\right\rangle$ state, to a final $\left|\Psi_{\epsilon_{i}}^{J M}\right\rangle$ state, with $J M$ the total momentum of the valence pair, by means of its interaction with a proton, with initial momentum $\vec{k}_{1}$ (final momentum 
$\left.\vec{k}_{1}^{\prime}\right)$ in the nucleon-nucleus center-of-mass frame, and initial $\sigma$ (final $\sigma^{\prime}$ ) spin projection. The proton momentum transferred is $\vec{\Delta}=\vec{k}_{1}^{\prime}-\vec{k}_{1}$.

In this case we have to consider the scattering from valence nucleons and a spin zero $\alpha$ core. Explicitly then for the scattering from valence nucleon $\mathcal{I}=2$

$$
\left\langle\vec{q}_{12}^{\prime}\left|t_{12}\left(\omega_{12}\right)\right| \vec{q}_{12}\right\rangle=\sum_{\kappa q a b} t_{\kappa q}^{(a b)}\left(\omega_{12}, \vec{q}_{12}^{\prime}, \vec{q}_{12}\right) \mathcal{T}_{\kappa q}^{\dagger}(a, b),
$$

with $0 \leqslant \kappa \leqslant 2$, and similarly for the scattering from valence nucleon $\mathcal{I}=3$. For the scattering from the spin zero core

$$
\left\langle\vec{q}_{14}^{\prime}\left|t_{14}\left(\omega_{14}\right)\right| \vec{q}_{14}\right\rangle=\sum_{a \alpha} t_{a \alpha}^{(a 0)}\left(\omega_{14}, \vec{q}_{14}^{\prime}, \vec{q}_{14}\right) \tau_{a \alpha}\left(s_{1} ; 1\right),
$$

with $0 \leqslant a \leqslant 1$. Within the impulse approximation the contribution to the scattering from one valence nucleon can be written as a sum in terms of spin transfer:

$$
\begin{aligned}
& \left\langle\vec{k}_{1}^{\prime} \chi_{s_{1}}^{\sigma^{\prime}} ; \varphi_{\epsilon_{i}}^{J M}\left|\hat{t}_{12}\right| \vec{k}_{1} \chi_{s_{1}}^{\sigma} ; \varphi_{0}^{J_{0} M_{0}}\right\rangle \\
& \quad=\sum_{b \beta} t_{\left[b \beta \mathcal{S}_{p} \mathcal{S}_{p}^{\prime}\right]}\left(\omega_{12}, \Delta\right) \times \rho_{\left[b \beta ; \mathcal{S}_{T} \mathcal{S}_{T}^{\prime} \epsilon_{i}\right]}\left(\frac{m_{3}}{M_{23}} \vec{\Delta}, \frac{m_{4}}{M_{234}} \vec{\Delta}\right),
\end{aligned}
$$

where $\mathcal{S}_{p}=\left\{s_{1} \sigma\right\}\left(\mathcal{S}_{p}^{\prime}=\left\{s_{1} \sigma^{\prime}\right\}\right)$ are the incoming (outgoing) spin of the nucleon and its projection and, $\mathcal{S}_{T}=\left\{J_{0} M_{0}\right\}\left(\mathcal{S}_{T}^{\prime}=\right.$ $\{J M\}$ ) the incoming (outgoing) total spin of the halo valence pair. $M_{23}=m_{2}+m_{3}, M_{234}=m_{2}+m_{3}+m_{4}$. The amplitude $t_{\left[b \beta \mathcal{S}_{p} \mathcal{S}_{p}^{\prime}\right]}$ is given in terms of the tensor components of the nucleon-nucleon transition amplitude as

$$
\begin{aligned}
t_{\left[b \beta \mathcal{S}_{p} \mathcal{S}_{p}^{\prime}\right]}\left(\omega_{12}, \Delta\right)= & \sum_{\kappa q a \alpha}(-)^{q} \frac{\hat{s}_{2}}{\hat{b}}\left\langle s_{1}|| \tau_{a}\left(\frac{1}{2} ; 1\right)|| s_{1}\right\rangle \\
& \times\left\langle s_{2}|| \tau_{b}\left(\frac{1}{2} ; 2\right)|| s_{2}\right\rangle\left(s_{1} \sigma a \alpha \mid s_{1} \sigma^{\prime}\right) \\
& \times(a \alpha b \beta \mid \kappa q) t_{\kappa q}^{(a b)}\left(\omega_{12}, \Delta\right) .
\end{aligned}
$$

The appropriate energy parameter is

$$
\omega_{12}=\frac{\mu_{12}}{\mu_{1,234}} E,
$$

with $\mu_{12}=m_{1} m_{2} /\left(m_{1}+m_{2}\right), \mu_{1,234}=m_{1} M_{234} /\left(m_{1}+M_{234}\right)$. The density form factor is given as a sum in terms of $d$ the total spin transfer $\Delta J$, and $c$ the angular momentum transfer $\Delta \ell_{y}$,

$\rho_{\left[b \beta ; \mathcal{S}_{T} \mathcal{S}_{T}^{\prime} \epsilon_{i}\right]}\left(\frac{m_{3}}{M_{23}} \vec{\Delta}, \frac{m_{4}}{M_{234}} \vec{\Delta}\right)=\sum_{c d} \hat{\rho}_{[b d c]}^{J J_{0} \epsilon_{i}}\left(\frac{m_{3}}{M_{23}} \Delta, \frac{m_{4}}{M_{234}} \Delta\right)$

$$
\times \hat{\varrho}_{[b \beta d c]}^{J M J_{0} M_{0}}(\hat{\Delta})
$$

with $\hat{\rho}_{[b d c]}^{J J_{0} \epsilon_{i}}$ calculated from the radial wave functions of the valence halo pair

$$
\begin{aligned}
\hat{\rho}_{[b d c]}^{J J_{0} \epsilon_{i}}\left(\frac{m_{3}}{M_{23}} \Delta, \frac{m_{4}}{M_{234}} \Delta\right)= & \sum i^{-\ell-\ell^{\prime}} \mathcal{G} \int r^{2} d r R^{2} d R \\
& \times \widehat{F}_{\mathcal{K}^{\prime} \gamma^{\prime}}^{\epsilon_{i} J}(r, R) \widehat{F}_{\mathcal{K}^{\hat{K}_{\gamma}} J_{0}}^{\epsilon_{0}}(r, R) \\
& \times j_{\ell}\left(\frac{m_{3}}{M_{23}} \Delta r\right) j_{\ell^{\prime}}\left(\frac{m_{4}}{M_{234}} \Delta R\right)
\end{aligned}
$$

and

$$
\begin{aligned}
\hat{\varrho}_{[b \beta d c]}^{J M J_{0} M_{0}}(\hat{\Delta})= & \sum_{\delta \gamma}(-)^{\left(M+M_{0}-\delta\right)}(b \beta d \delta \mid c \gamma)\left(J M d \delta \mid J_{0} M_{0}\right) \\
& \times \sqrt{4 \pi} Y_{c \gamma}(\hat{\Delta})
\end{aligned}
$$

In Eq. (38) $\mathcal{G}$ is a geometric factor given by

$$
\begin{aligned}
\mathcal{G}= & (-)^{J+J_{0}+s_{2}-s_{3}+L+L^{\prime}+\ell+\ell^{\prime}+b+c+d} \\
& (-)^{2\left(S+S^{\prime}-\ell_{y}^{\prime}-\ell_{x}-\gamma+d-b\right)} \frac{\hat{\ell}^{2} \hat{\ell}^{\prime 2} \hat{d}^{2} \hat{b} \hat{S} \hat{S}^{\prime} \hat{\ell_{x}} \hat{\ell_{y}} \hat{L} \hat{L}^{\prime} \hat{J}}{\hat{c}} \\
& \times W\left(S s_{2} S^{\prime} s_{2} ; s_{3} b\right) \\
& \times\left(\ell 0 \ell_{x} 0 \mid \ell_{x}^{\prime} 0\right)\left(\ell 0 \ell^{\prime} 0 \mid c 0\right)\left(\ell^{\prime} 0 \ell_{y} 0 \mid \ell_{y}^{\prime} 0\right) \\
& \times\left\{\begin{array}{ccc}
b & d & c \\
S^{\prime} & J & L^{\prime} \\
S & J_{0} & L
\end{array}\right\}\left\{\begin{array}{ccc}
\ell & \ell^{\prime} & c \\
\ell_{x} & \ell_{y} & L \\
\ell_{x}^{\prime} & \ell_{y}^{\prime} & L^{\prime}
\end{array}\right\} .
\end{aligned}
$$

The following triangle relations follow from the geometric coefficients: $\left\{\ell_{y}^{\prime}, \ell_{y}, c\right\},\left\{J, J_{0}, d\right\},\left\{S^{\prime}, S, b\right\}$. The scattering from the core assumed here as spinless can equivalently be written as

$$
\begin{aligned}
\left\langle\vec{k}_{1}^{\prime} \chi_{s_{1}}^{\sigma^{\prime}} ; \varphi_{\epsilon_{i}}^{J M}\left|\hat{t}_{14}\right| \vec{k}_{1} \chi_{S_{1}}^{\sigma} ; \varphi_{0}^{J_{0} M_{0}}\right\rangle= & t_{\left[00 \mathcal{S}_{p} \mathcal{S}_{p}^{\prime}\right]}\left(\omega_{14}, \Delta\right) \\
& \times \rho_{\left[00 ; \mathcal{S}_{T} \mathcal{S}_{T}^{\prime} \epsilon_{i}\right]}\left(0, \frac{M_{23}}{M_{234}} \vec{\Delta}\right) .
\end{aligned}
$$

The amplitude $\hat{t}_{\left[00 \mathcal{S}_{p} \mathcal{S}_{p}^{\prime}\right]}$ is given in terms of the tensor components of the nucleon-nucleon transition amplitude as

$$
\begin{aligned}
t_{\left[00 \mathcal{S}_{p} \mathcal{S}_{p}^{\prime}\right]}\left(\omega_{14}, \Delta\right)= & \sum_{a \alpha}(-)^{\alpha}\left\langle s_{1}|| \tau_{a}\left(\frac{1}{2} ; 1\right)|| s_{1}\right\rangle \\
& \times\left(s_{1} \sigma a \alpha \mid s_{1} \sigma^{\prime}\right) t_{a \alpha}^{(a 0)}\left(\omega_{14}, \Delta\right) .
\end{aligned}
$$

The appropriate energy parameter is similarly given as

$$
\omega_{14}=\frac{\mu_{14}}{\mu_{1,234}} E
$$

The density form factor is

$$
\begin{aligned}
\rho_{\left[00 ; \mathcal{S}_{T} \mathcal{S}_{T}^{\prime} \epsilon_{i}\right]}\left(0, \frac{M_{23}}{M_{234}} \vec{\Delta}\right)= & \sum_{c} \hat{\rho}_{[0 c c]}^{J J_{0} \epsilon_{i}}\left(0, \frac{M_{23}}{M_{234}} \Delta\right) \\
& \times \hat{\varrho}_{[00 c c]}^{J M J_{0} M_{0}}(\hat{\Delta}) .
\end{aligned}
$$

The cross section for the transition to each pseudostate of associated energy $\epsilon_{i}$ is evaluated from the transition matrix elements according to

$$
\begin{aligned}
\frac{d \sigma_{J J_{0}}}{d \Omega}\left(\epsilon_{i}\right)= & \frac{1}{\left(\widehat{s_{1}}\right)^{2}} \frac{1}{\left(\widehat{J_{0}}\right)^{2}}\left[\frac{\hbar^{2}}{4 \pi^{2} \mu_{1 A}}\right]^{2} \sum_{\sigma \sigma^{\prime}} \sum_{M M_{0}} \\
& \times\left|\left\langle\vec{k}_{1}^{\prime} \chi_{s_{1}}^{\sigma^{\prime}} ; \varphi_{\epsilon_{i}}^{J M}\left|\sum_{\mathcal{I}} \hat{t}_{1 \mathcal{I}}\right| \vec{k}_{1} \chi_{s_{1}}^{\sigma} ; \varphi_{0}^{J_{0} M_{0}}\right\rangle\right|^{2} .
\end{aligned}
$$

In order to get a continuum distribution for the double cross section it must be convoluted with the associated widths:

$$
\frac{d^{2} \sigma_{J J_{0}}}{d \Omega d E}=\sum_{\epsilon_{i}} f\left(E, \epsilon_{i}, \Gamma^{J \epsilon_{i}}\right) \frac{d \sigma_{J J_{0}}}{d \Omega}\left(\epsilon_{i}\right) .
$$




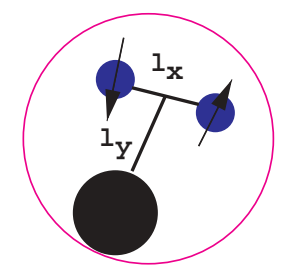

FIG. 1. (Color online) ${ }^{6} \mathrm{He}$ Borromean nucleus.

For the convolution kernels we use a Gaussian function

$$
f_{\text {gauss }}\left(E, \epsilon_{i}, \Gamma^{\hat{J} \epsilon_{i}}\right)=\frac{1}{\sqrt{\pi} \Gamma^{J \epsilon_{i}}} \exp \left[-\left(\left(E-\epsilon_{i}\right) / \Gamma^{\hat{J} \epsilon_{i}}\right)^{2}\right]
$$

with the widths taken from Eq. (22).

\section{RESULTS}

As discussed in Sec. II, according to MST, one needs the matrix elements for the transition amplitude that describe the scattering from the valence neutrons and the $\alpha$ core. The former is obtained from a realistic $N N$ Paris interaction. The transition amplitude for proton scattering from the ${ }^{4} \mathrm{He}$ core was generated from fitting the differential cross section elastic scattering data $p+{ }^{4} \mathrm{He}$ at $E_{\mathrm{p}}=700 \mathrm{MeV}[25,26]$ and $800 \mathrm{MeV}$ [27] shown in Fig. 2(a). We have taken a phenomenological potential of Woods-Saxon (WS) shape, i.e.,

$$
U(r)=V_{0} f\left(r, r_{0}, a_{0}\right)+i W_{i} f\left(r, r_{i}, a_{i}\right)
$$
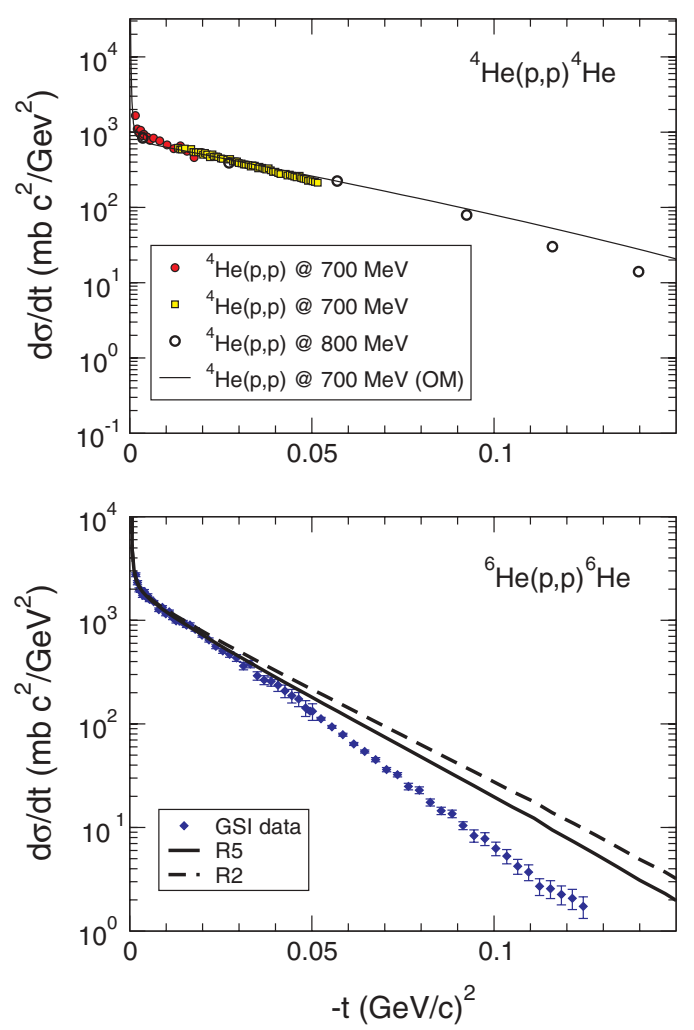

FIG. 2. (Color online) Elastic differential cross section for $p-{ }^{4,6} \mathrm{He}$ at $700 \mathrm{MeV}$. In the upper figure, the solid line represents an optical model fit of the data. with $f\left(r, r_{x}, a_{x}\right)=\left[1+\exp \left[\left(r-R_{x}\right) / a_{x}\right]^{-1}\right.$, where $R_{x}=$ $r_{x} A^{1 / 3}$. The best-fit analysis of the data yields the values $V_{0}=+72 \mathrm{MeV}, W_{i}=-115 \mathrm{MeV}, r_{0}=r_{i}=1.14 \mathrm{fm}$, and $a_{0}=a_{i}=0.31 \mathrm{fm}[13]$.

The elastic differential cross section for $p-{ }^{6} \mathrm{He}$ shown in Fig. 2(b) reproduces reasonably well the scattering data up to $15^{\circ}\left(-t=\Delta^{2}=k_{0}^{2}(1-\cos \theta)=0.0657(\mathrm{GeV} / c)^{2}\right)$. At larger scattering angles higher order multiple scattering terms become important in the description of the elastic scattering [28]. The calculated cross section using the R2 model (dashed line) decays slightly slower than the observable calculated using the R5 model. This is expected since the rms of ${ }^{6} \mathrm{He}$ obtained from the R2 model is smaller than the one obtained from the R5 model. Also within a simplified shakeoff picture we expect that the single scattering approximation underestimates the breakup cross section at very large momentum transfer. Nevertheless, it will give us an indication of what type of excitations are relevant at this high energy regime.

Within the MST scattering framework the double differential cross section $d^{2} \sigma / d \Omega d E$ for all final state contributions can be evaluated explicitly after convolution with the associated widths. If integration is made over the angular acceptance of the proton telescopes, the energy spectrum $d \sigma / d E$ is obtained; on the other hand, the angular distribution of a particular final state can be singled out. We shall separately analyze the two cases.

The calculated contributions from each final state to the inelastic differential cross sections for $p-{ }^{6} \mathrm{He}$ using the R2 (light lines) and R5 (dark lines) models are shown in Fig. 3. The nonresonant dipole excitation $J^{\pi}=1^{-}$(dashed line) is the dominant contribution between $10^{\circ}$ and $20^{\circ}$ in agreement with Ref. [9]. The resonant excitation $J^{\pi}=2^{+}$(thin solid line) becomes significant at larger angles. There is also some contribution from the $J^{\pi}=0_{2}^{+}$transition (dotted line). In addition, the contribution from the $J^{\pi}=1^{+}$excitation is comparatively much smaller and that of the $J^{\pi}=0^{-}$

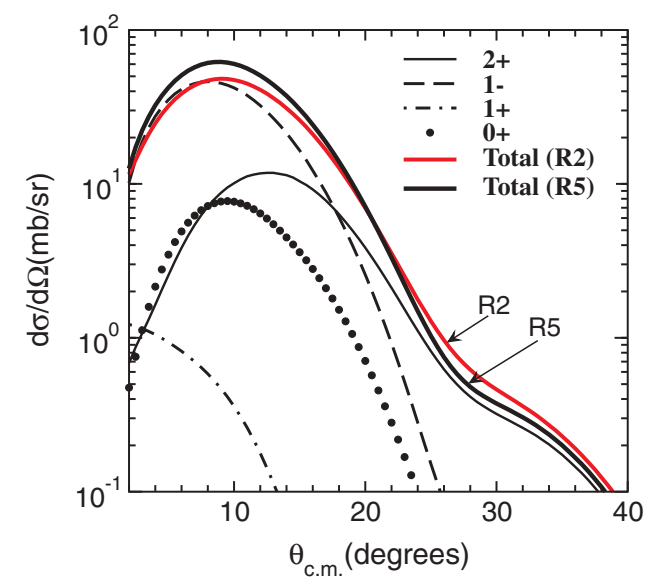

FIG. 3. (Color online) Calculated contributions for the inelastic differential cross section at $700 \mathrm{MeV}$ for the final states $J^{\pi}=$ $2^{+}$(thin solid line), $J^{\pi}=1^{-}$(dashed line), $J^{\pi}=1^{+}$(dashed dotted), $J^{\pi}=0_{2}^{+}$(dotted), $J^{\pi}=0^{-}$(dashed double dotted) using the R2 model. The thick lines represent the sum of all contributions for the R2 and R5 models. 


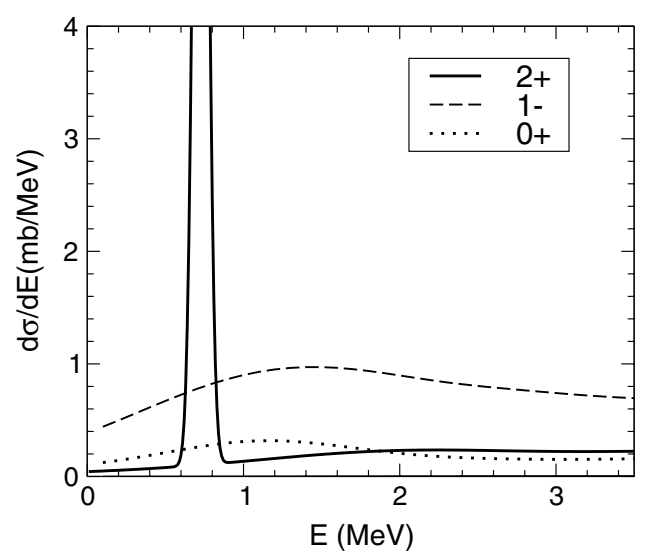

FIG. 4. Calculated energy spectrum for $p-{ }^{6} \mathrm{He}$ inelastic scattering at $700 \mathrm{MeV}$.

excitation negligible. This shows that spin flip transitions give a small contribution to the scattering at high energies. In fact, spin-flip transitions are only significant in forward scattering and high energy scattering probes large momentum transferred.

The sum of all transitions to final resonant/nonresonant states is also shown in Fig. 3. The R2 model (light thick solid line) predicts a slightly broader inelastic cross section than the R5 model (dark thick solid line).

For the purpose of analysing the energy spectrum we integrate the angular distribution up to $60^{\circ}$. Figure 4 shows the $J^{\pi}=2^{+}$energy excitation (solid line) which is a true resonance with a narrow peak at $\mathrm{E}^{*}=\epsilon_{i}+\epsilon_{0}=1.7 \mathrm{MeV}$. Thus the wave function and therefore the transition has a Breit-Wigner form in all the components Eq. (27). We can also see some low lying strength accumulation background for the transition to the $J^{\pi}=1^{-}$state (dashed line). This is due to the strong nn attraction in the relative $\mathrm{S}$-wave. The contribution from the $J^{\pi}=0^{+}$state (dotted line) is very small.

\section{CONCLUSIONS}

We have shown detailed formal expressions for the MST multiple scattering expansion formalism within the single scattering approximation applied to inelastic excitations. As an advantage to other scattering frameworks that can be found in the literature the spin nucleon-nucleon interactions are included.

The low-lying ${ }^{6} \mathrm{He}$ continuum was generated using the hyperspherical harmonics pseudostate method. A GaussLaguerre square integrable basis was used in the expansion of the wave function for the excited states.

This formalism was used to study proton inelastic scattering from ${ }^{6} \mathrm{He}$ at $700 \mathrm{MeV}$. We have found that the dominant contribution to the inelastic cross section is the dipole transition $0^{+} \rightarrow 1^{-}$. Significant contributions from the resonant excitation $0^{+} \rightarrow 2^{+}$at larger angles and also from the non resonant excitation $0^{+} \rightarrow 0_{2}^{+}$are found. The cross section is therefore a complex mixture of several excitations at this energy. We have also shown that the spin flip transitions give a small contribution to the scattering at high energies.

\section{ACKNOWLEDGMENTS}

This work was supported by Fundação para a Ciência e Tecnologia (Portugal) through grant No. POCTI/FNU/43421/2001, Acção Integrada Luso-Espanhola E-75/04 and in the U.K. by EPSRC grant GR/T/28577. A.M.M. acknowledges the financial support of the Junta de Andalucía.
[1] Y. Alhassid, M. Gai, and G. F. Bertsch, Phys. Rev. Lett. 49, 1482 (1982).

[2] A. Cobis, D. V. Fedorov, and A. S. Jensen, Phys. Rev. C 58, 1403 (1998).

[3] I. J. Thompson et al., J. Phys. G 24, 1505 (1998).

[4] A. A. Korsheninnikov et al., Nucl. Phys. A616, 189c (1997).

[5] M. V. Zhukov et al., Phys. Rep. 4, 151 (1993).

[6] S. Nakayama et al., Phys. Rev. Lett. 85, 262 (2000).

[7] R. Crespo, I. J. Thompson, and A. A. Korsheninnikov, Phys. Rev. C 66, 021002(R) (2002).

[8] A. A. Korsheninnikov et al., Phys. Rev. Lett. 78, 2317 (1997).

[9] S. N. Ershov, T. Rogde, B. V. Danilin, J. S. Vaagen, I. J. Thompson, and F. A. Gareev, Phys. Rev. C 56, 1483 (1997).

[10] S. N. Ershov, B. V. Danilin, and J. S. Vaagen, Phys. Rev. C 64, 064609 (2001).

[11] S. N. Ershov, B. V. Danilin, and J. S. Vaagen, Phys. Rev. C 62, 041001(R) (2000).

[12] R. Crespo and R. C. Johnson, Phys. Rev. C 60, 034007 (1999).

[13] R. Crespo, A. M. Moro, and I. J. Thompson, Nucl. Phys. A771, 26 (2006).

[14] R. Crespo, A. M. Moro, and I. J. Thompson, Phys. At. Nucl. 69, 1254 (2006).
[15] R. Crespo and I. J. Thompson, Phys. Rev. C 63, 044003 (2001).

[16] B. V. Danilin, I. J. Thompson, M. V. Zhukov, and J. S. Vaagen, Nucl. Phys. A632, 383 (1998).

[17] I. J. Thompson, Comput. Phys. Rep. 7, 167 (1988).

[18] I. J. Thompson, B. V. Danilin, V. D. Efros, J. S. Vaagen, J. M. Bang, and M. V. Zhukov, Phys. Rev. C 61, 24318 (2000).

[19] V. P. Bugrov and S. G. Kadmensky, Yad. Fiz. 49, 1562 (1989) [Sov. J. Nucl. Phys. 49, 967 (1989)].

[20] S. G. Kadmensky and V. P. Bugrov, Yad. Fiz. 59, 424 (1996) [Phys. At. Nucl. 59, 399 (1996)].

[21] D. Gogny et al., Phys. Lett. B32, 591 (1970).

[22] J. Bang and C. Gignoux, Nucl. Phys. A313, 119 (1979).

[23] N. K. Timofeyuk and I. J. Thompson, Phys. Rev. C 61, 044608 (2000).

[24] R. Crespo and A. M. Moro, Phys. Rev. C 65, 054001 (2002).

[25] G. D. Alkhazov et al., Phys. Rev. Lett. 78, 2313 (1997).

[26] O. G. Grebenjuk et al., Nucl. Phys. A500, 637 (1989).

[27] L. G. Arnold, B. C. Clark, and R. L. Mercer, Phys. Rev. C 19, 917 (1979).

[28] J. Al-Khalili, R. Crespo, A. M. Moro, and I. J. Thompson (in preparation). 